\title{
Constraints on wave drag parameterization schemes for simulating the quasi-biennial oscillation. Part I: gravity wave forcing
}

Article

Published Version

Campbell, L. J. and Shepherd, T. G. (2005) Constraints on wave drag parameterization schemes for simulating the quasibiennial oscillation. Part I: gravity wave forcing. Journal of the Atmospheric Sciences, 62 (12). pp. 4178-4195. ISSN 15200469 doi: https://doi.org/10.1175/JAS3616.1 Available at https://centaur.reading.ac.uk/32099/

It is advisable to refer to the publisher's version if you intend to cite from the work. See Guidance on citing.

To link to this article DOI: http://dx.doi.org/10.1175/JAS3616.1

Publisher: American Meteorological Society

All outputs in CentAUR are protected by Intellectual Property Rights law, including copyright law. Copyright and IPR is retained by the creators or other copyright holders. Terms and conditions for use of this material are defined in the End User Agreement. 


\section{CentAUR}

Central Archive at the University of Reading

Reading's research outputs online 


\title{
Constraints on Wave Drag Parameterization Schemes for Simulating the Quasi-Biennial Oscillation. Part I: Gravity Wave Forcing
}

\author{
Lucy J. CAmpbell* and Theodore G. Shepherd \\ Department of Physics, University of Toronto, Toronto, Ontario, Canada
}

(Manuscript received 28 April 2004, in final form 14 June 2005)

\begin{abstract}
Parameterization schemes for the drag due to atmospheric gravity waves are discussed and compared in the context of a simple one-dimensional model of the quasi-biennial oscillation (QBO). A number of fundamental issues are examined in detail, with the goal of providing a better understanding of the mechanism by which gravity wave drag can produce an equatorial zonal wind oscillation. The gravity wave-driven QBOs are compared with those obtained from a parameterization of equatorial planetary waves. In all gravity wave cases, it is seen that the inclusion of vertical diffusion is crucial for the descent of the shear zones and the development of the QBO. An important difference between the schemes for the two types of waves is that in the case of equatorial planetary waves, vertical diffusion is needed only at the lowest levels, while for the gravity wave drag schemes it must be included at all levels. The question of whether there is downward propagation of influence in the simulated QBOs is addressed. In the gravity wave drag schemes, the evolution of the wind at a given level depends on the wind above, as well as on the wind below. This is in contrast to the parameterization for the equatorial planetary waves in which there is downward propagation of phase only. The stability of a zero-wind initial state is examined, and it is determined that a small perturbation to such a state will amplify with time to the extent that a zonal wind oscillation is permitted.
\end{abstract}

\section{Introduction}

Measurements of the zonal wind in the equatorial stratosphere over the past $50 \mathrm{yr}$ show that there is an oscillation in wind direction characterized by alternating easterly and westerly phases that descend with time (Ebdon 1960; Reed et al. 1961). Since the period of the oscillation is $26-30$ months, it has come to be known as the quasi-biennial oscillation (QBO). A review of the discovery of the QBO is given by Baldwin et al. (2001), who also give an overview of what is currently known about it.

It is well known that the QBO is driven by momentum transfer from waves propagating upward from the troposphere. However, there has been considerable de-

\footnotetext{
* Current affiliation: School of Mathematics and Statistics, Carleton University, Ottawa, Ontario, Canada.
}

Corresponding author address: Dr. T. G. Shepherd, Dept. of Physics, University of Toronto, Toronto, ON M5S 1A7, Canada. E-mail: tgs@atmosp.physics.utoronto.ca bate as to the relative contributions of the different types of waves involved. Lindzen and Holton (1968) initially postulated that the QBO is driven by internal gravity waves that interact with the mean flow through critical level absorption. In their model, there is a continuous spectrum of upward-propagating waves, each with a different critical level, and oscillations in the mean wind result on specifying a suitable upper boundary condition to simulate a stratopause semiannual oscillation (SAO). Holton and Lindzen (1972, hereafter HL72) later put forward a revised theory for the QBO based instead on absorption of equatorial planetary waves by thermal damping. They outlined the mechanism that leads to the downward propagation of the shear zones, and noted that vertical diffusion provides a means for switching between easterly and westerly phases at low levels. Plumb (1977) reexamined the model of HL72 and elucidated the mechanism for the shear layer descent by means of a linear stability analysis. Plumb showed that, in the HL72 model, the descent of the shear zones is only a downward propagation of phase, not of information; in the inviscid limit, the evolution of the wind at a given level is independent of that 
at higher levels. He also explained in detail the effect of vertical diffusion and its role in the switching mechanism at low levels. The HL72 model of the QBO thereafter became the accepted paradigm (Andrews et al. 1987).

However, until recently, large-scale atmospheric general circulation models (GCMs) were unable to simulate the QBO. This inability was seen as a notable failing of the models, as well as something of a puzzle. Equatorial planetary waves are simulated explicitly by GCMs and thermal damping is represented, so the conditions for a QBO are satisfied according to the HL72 theory. Dunkerton (1997) provided an explanation for the lack of accurate QBO simulations by GCMs. He observed that the Brewer-Dobson upwelling in the lower stratosphere acts to suppress the oscillation in the mean wind. With realistic upwelling, not included in the models of HL72 or Plumb (1977), observed equatorial planetary wave amplitudes are apparently insufficient to drive a QBO. This observation was supported by the two-dimensional simulations of the QBO by Gray and Pyle (1989), which required equatorial planetary wave amplitudes approximately 3 times greater than observed amplitudes. Dunkerton (1997) argued that the additional wave drag needed to drive the QBO must be provided by gravity waves that, because of their small spatial scale, are generally unresolved in GCMs.

Around the time of Dunkerton's (1997) article, GCM simulations of the QBO began to emerge. Those GCMs that did simulate QBOs tended either to be of very high vertical resolution [Takahashi (1996) and Horinouchi and Yoden (1998) with simplified GCMs, Hamilton et al. (1999) with the high-resolution SKYHI GCM] or to include a parameterized gravity wave drag scheme (Scaife et al. 2000; Giorgetta et al. 2002; McLandress 2002), typically with gravity wave amplitudes significantly enhanced in the Tropics. This evidence suggests that Dunkerton (1997) is correct in hypothesizing that gravity wave drag is needed to drive a QBO in the earth's atmosphere, given the observed strength of the equatorial planetary waves. Unfortunately, there is a lack of quantitative information from observations to infer constraints on gravity wave parameters for use in models. In particular, the specification of the spectrum of gravity waves at their source remains a major uncertainty.

It must however be emphasized that GCMs that do simulate QBOs seem to do so for various reasons, and it is possible that a QBO can be obtained for the wrong reasons by simply tuning the model parameters. In general, the characteristics of the QBOs generated by GCMs and the requirements to obtain these oscillations are different for each model. This is not surprising, given that the form of the simulated QBO depends on a number of factors, many of which are modeldependent. For example, a GCM with overactive convective adjustment might generate overly strong equatorial planetary waves (Horinouchi et al. 2003) and hence require less or even no parameterized gravity wave drag to be able to generate a QBO. Furthermore, McLandress (2000, poster presentation: P/1-6.10 "Equatorial Oscillations in a Middle Atmosphere GCM" at the Second General Assembly of the WCRP SPARC Project, 6-10 November 2000) showed that the choice of finite-difference method used for calculating the divergence of the gravity wave flux can have a dramatic effect on the drag profile and hence on the period and other characteristics of the oscillation.

All these considerations suggest that there is a need for an understanding of the properties of gravity wave drag parameterization schemes in the context of a simulated QBO. In this paper, we take a first step toward this goal by examining the characteristics of QBOs forced by parameterized gravity wave drag in a simple one-dimensional zonal mean model of the equatorial zonal wind. While the case of pure gravity wave drag is not directly relevant to the terrestrial QBO, it is an interesting problem in wave, mean-flow interaction in its own right. Moreover, it serves as a basis for addressing the more realistic case of gravity wave drag plus equatorial planetary wave drag, which is the focus of a companion paper. The gravity wave drag parameterization schemes used in our study are well-known schemes that are based on the theory of wave breaking and saturation (Lindzen 1981). The characteristics of the schemes are compared with those of the HL72 parameterization scheme for equatorial planetary wave drag with respect to their ability to simulate a QBO.

Our investigation is aimed at providing answers to the following questions. For a given parameterization scheme, what are the conditions on the initial configuration and the choice of parameters for the mean wind to evolve to an oscillating state? Could a zero initial velocity profile ever be a stable equilibrium state or would a small perturbation to a zero velocity state eventually grow large enough to allow the development of a QBO? What is the mechanism for the descent of the shear zones? What factors affect the form and, in particular, the period of the oscillation (assuming one is possible)? What role does diffusion play, in particular in the mechanism for switching between easterly and westerly winds at the lowest levels? In the HL72 scheme, it is necessary to include vertical diffusion in a layer above the source level of the waves; in the gravity wave drag schemes is the inclusion of diffusion at low levels a sufficient requirement to generate a $\mathrm{QBO}$, or is 
diffusion needed at all levels? Is there downward or upward propagation of information, that is, does the evolution of the wind at a given level depend on the wind above or below?

In the HL72 scheme, the wave drag at a given level depends on the cumulative effect of the wind below. In the gravity wave drag schemes, the mathematical expression for the drag due to a wave of a given phase speed at a given level depends on the local flow conditions. However, the total drag (corresponding to all the waves in the spectrum) at each level does depend on the wind below, albeit in a more subtle way than in the HL72 scheme. For a parameterization to be able to generate a spontaneous QBO, the net drag corresponding to a given wind speed at a given level must take both signs over each oscillation period. But that is not possible if the drag is a single-valued function of the local wind speed. It is the dependence of the total drag on the flow conditions below the level in question that allows the gravity wave drag schemes to generate QBOs.

In seeking answers to the questions posed in the preceding paragraphs, we derive the constraints that are needed for each scheme to be able to generate a QBOlike oscillation, that is, an oscillation between easterly and westerly zonal mean winds that resembles the QBO in at least the following respects: (i) its period is within a range of about 700-900 days, (ii) its maximum amplitude tends to a steady value within the range of velocities $20-50 \mathrm{~m} \mathrm{~s}^{-1}$, and (iii) it takes place over a range of heights from the source level up to at least 50 $\mathrm{km}$. The choice of $50 \mathrm{~km}$ as the minimum upper level for the QBO winds was simply to ensure that the QBO took place over a wide altitude range roughly corresponding to the equatorial stratosphere; however, since the model used in our simulations does not possess a stratopause, this choice was quite arbitrary and any other level could have been selected. The constraints derived in our study include restrictions on the choice of the relevant parameters, on the initial configuration and, where appropriate, on the gravity wave source spectrum.

A number of the issues raised above were addressed by Plumb (1977) for the HL72 scheme for equatorial planetary waves, and in the next section we give a summary of his most important results. In section 3, Lindzen's gravity wave drag parameterization is examined for the case in which the forcing consists of only two waves, and in section 4 we examine Alexander and Dunkerton's (1999) variant of the Lindzen scheme. In section 5, Lindzen's scheme is generalized to include a continuous spectrum of waves over a range of phase speeds. Finally, a discussion and comparison of the different schemes is presented in section 6 .

\section{The HL72 parameterization of equatorial planetary waves}

The one-dimensional model used in our QBO simulations comprises the single equation

$$
\frac{\partial \bar{u}}{\partial t}-\nu \frac{\partial^{2} \bar{u}}{\partial z^{2}}=X(z, t),
$$

where $\bar{u}(z, t)$ is the zonally averaged zonal velocity, $X$ $(z, t)$ is the forcing from equatorial planetary waves and/or gravity waves, and $\nu$ is the vertical diffusivity. The equation is solved numerically in a domain extending from a height of $15 \mathrm{~km}$ (around the equatorial tropopause) up to a height of $100 \mathrm{~km}$. The finitedifference scheme used for the numerical solution of the equation is second-order accurate in space and in time. The source level of the waves is taken to be the lower boundary of the model. For comparison with the gravity wave drag experiments that are described in sections 3-5, some QBO simulations were carried out with planetary wave drag calculated using the HL72 scheme. In this section, we present some results of those simulations and give an overview of the characteristics of the HL72 scheme, as discussed by Plumb (1977).

Plumb's discussion of the HL72 theory focused on the configuration involving a westerly Kelvin wave and an anti-Kelvin wave. The latter is an easterly wave that does not exist in nature; it is assumed to be unaffected by the earth's rotation and thus has the same form as a Kelvin wave except that its phase speed and momentum flux are in the opposite direction. [The term antiKelvin wave was suggested by Dunkerton (1991).] The advantage of using such a wave instead of a mixed Rossby-gravity wave is that the two waves then have identical expressions for their drags:

$$
\begin{aligned}
X^{ \pm}(z, t)= & \frac{N \mu e^{-z_{0} / H} F_{0}^{ \pm}}{k^{ \pm}\left[\bar{u}(z, t)-c^{ \pm}\right]^{2} \bar{\rho}_{0}} \\
& \times \exp \left\{-\int_{z_{0}}^{z} \frac{N \mu}{k^{ \pm}\left[\bar{u}\left(z^{\prime}, t\right)-c^{ \pm}\right]^{2}} d z^{\prime}+\frac{z}{H}\right\},
\end{aligned}
$$

where the source level of the waves is $z=z_{0}$ and $F_{0}^{ \pm}$are the momentum fluxes, which are specified at the source level. The phase speeds and horizontal wave numbers of the waves are denoted by $c^{ \pm}$and $k^{ \pm}$, respectively and, in all cases, the plus sign refers to the westerly 
wave and the minus sign to the easterly wave. The other parameters are the Newtonian cooling rate $\mu$, the Brunt-Väisälä frequency $N$, and the density scale height $H$. The background density is assumed to be of the form $\bar{\rho}(z)=\bar{\rho}_{0} \exp \left[-\left(z-z_{0}\right) / H\right]$, where $\bar{\rho}_{0}$ is the density at the source level.

In a configuration where $k^{-}=k^{+}, c^{-}=-c^{+}$and $F_{0}^{-}=-F_{0}^{+}$, the initial mean wind must be nonzero initially in order for the total drag from the two waves to be nonzero. However, although $\bar{u}=0$ is a steady solution, it is unstable and a QBO will develop from any initial condition, if the parameters allow a QBO to exist (Plumb 1977). From (2.2), it is evident that the strength of the drag and, hence, the period of the oscillation, is determined by the magnitude of $F_{0}$ and by the ratio $N \mu H /\left(k c^{2}\right)$. This ratio also controls the rate of exponential growth or decay of the drag with height. An important conclusion of Plumb's (1977) analysis of this scheme is that in the absence of diffusion, there is no downward propagation of information. This means that imposing an oscillating upper boundary condition, as HL72 had done, has little effect on the evolution of the mean flow. Plumb observed that in the HL72 parameterization, the downward motion of the shear zones depends on the flow evolution at lower levels, which is controlled by the vertical diffusion at the lowest levels. Thus, diffusion at the lowest levels affects the period of the oscillation, but it is not needed at higher levels. Plumb also pointed out that in the case of Boussinesq dynamics [where the exp $(z / H)$ factor in (2.2) is neglected], the inviscid limit is singular. While diffusion is needed for switching between easterly and westerly flow, the period of the oscillation is independent of $\nu$, the diffusion parameter in (2.1), in the limit $\nu \rightarrow 0$; but if $\nu=0$, the period is infinite and a steady state is attained. In the case of exponentially varying density, on the other hand, the period of the oscillation increases and tends to infinity as $\nu \rightarrow 0$.

The abovementioned characteristics of the HL72 scheme apply also in the more realistic configuration where the anti-Kelvin wave is replaced by a mixed Rossby-gravity wave. In the expressions for the momentum flux and the drag due to the mixed Rossbygravity wave, the integrand in (2.2) is multiplied by a factor of $\left\{\beta /\left[k^{2}\left(\bar{u}-c^{-}\right)\right]-1\right\}$, where $\beta$ is the latitudinal gradient of planetary vorticity. This results in an asymmetry between the easterly and westerly regimes of the QBO.

Figure 1 shows the results of a QBO simulation using the HL72 scheme with the Kelvin/anti-Kelvin wave configuration. Here and in the rest of the paper, we set the density-scale height $H$ to $7 \mathrm{~km}$ and assume a con-

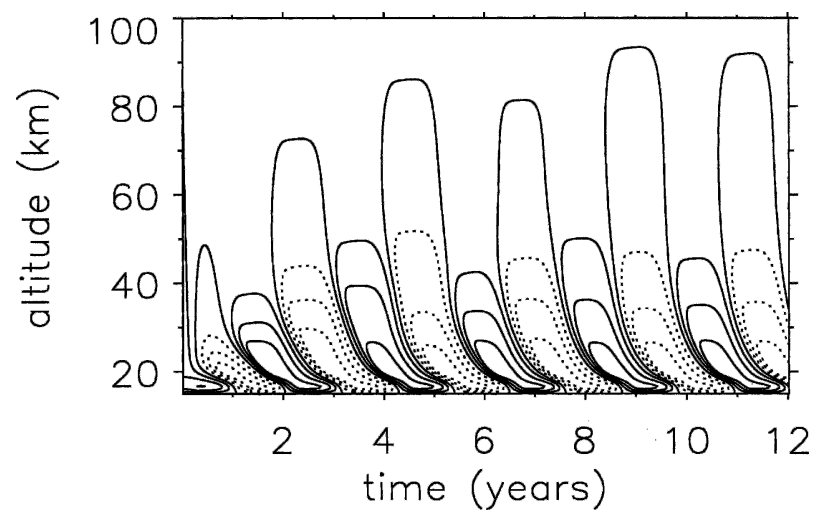

FIG. 1. Time-height plot of the zonal mean wind in a QBO simulation with the Kelvin and anti-Kelvin wave configuration. The solid contours denote westerlies, including the zero-wind line, and the dotted contours denote easterlies. Contour intervals are $5 \mathrm{~m} \mathrm{~s}^{-1}$.

stant Brunt-Väisälä frequency $N=0.02 \mathrm{~s}^{-1}$. The choice of wave parameters

$$
\begin{aligned}
F_{0}\left(c^{ \pm}\right) / \rho_{0} & =F_{0}^{ \pm} / \rho_{0}= \pm 7 \times 10^{-3} \mathrm{~m}^{2} \mathrm{~s}^{-2}, \\
c^{ \pm} & = \pm 25 \mathrm{~m} \mathrm{~s}^{-1}, \quad k^{ \pm}=2 \pi /\left(4 \times 10^{7} \mathrm{~m}\right),
\end{aligned}
$$

with $\mu=10^{-6} \mathrm{~s}^{-1}$ and $\nu=0.3 \mathrm{~m}^{2} \mathrm{~s}^{-1}$ gives a QBO with a period of about 26 months. The time-height contour plot of the zonal mean wind over a period of $12 \mathrm{yr}$ is shown for this configuration. The maximum wind amplitude is between 15 and $20 \mathrm{~m} \mathrm{~s}^{-1}$ and occurs within 10 $\mathrm{km}$ of the source level. The amplitude decreases with height because $N \mu /\left(k c^{2}\right)>1 / H$ and so the drag, as given by (2.2), decreases exponentially with height.

\section{Lindzen's parameterization with two waves}

Lindzen's theory of gravity wave breaking and saturation (Lindzen 1981; Holton 1982) was originally developed for a single wave, but it can be extended to the case of two or more noninteracting waves. To generate a QBO-like oscillation using this scheme, there must be at least two waves, one westerly and the other easterly, or a spectrum of waves over a range of both westerly and easterly phase speeds. The waves are assumed not to interact with each other, so that the total drag is simply the sum of the drags over the range of phase speeds in the spectrum. In this section, we examine the case where there are only two waves.

Each wave is assumed to propagate upward, its amplitude increasing with height, until it gets to a level, which we shall denote as $z_{b}$, where it becomes statically unstable and breaks. Using a Wentzel-KramersBrillouin (WKB) analysis (with the assumption that 
(a)

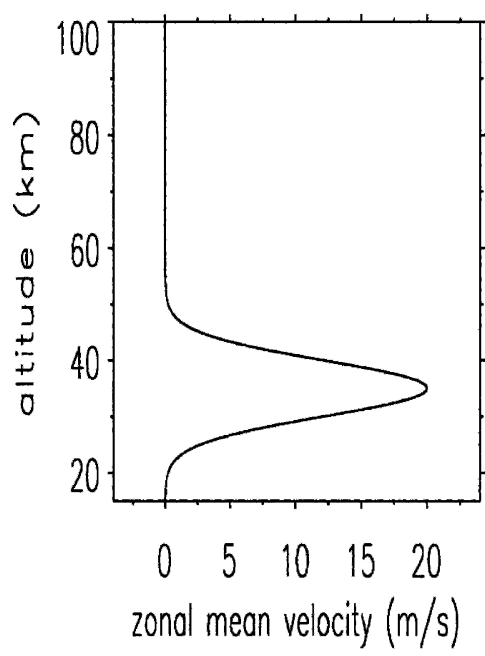

(b)

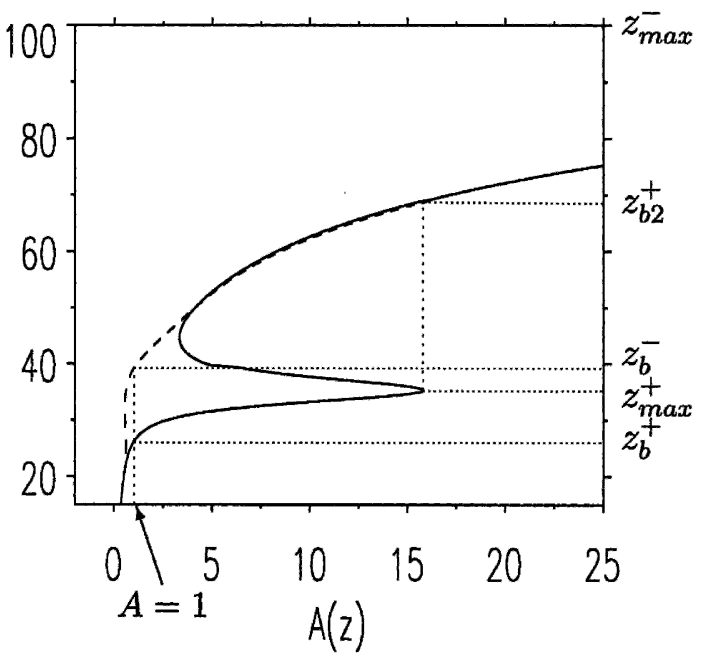

(c)

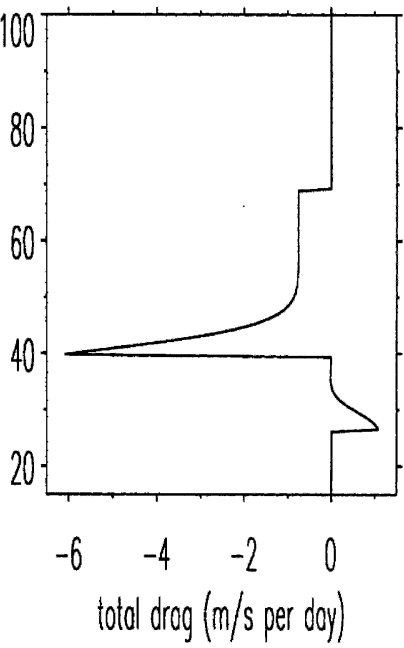

FIG. 2. Lindzen parameterization with two waves with phase speeds $c^{ \pm}= \pm 25 \mathrm{~m} \mathrm{~s}^{-1}$. (a) Initial mean velocity with maximum value of $\bar{u}_{\max }=20 \mathrm{~m} \mathrm{~s}^{-1}$, (b) the function $A^{ \pm}(z)$ defined in (3.1), and (c) the total drag from the two waves.

variations with time and height in the background flow are slow compared with such variations in the waves), Lindzen (1981) derived the following criterion for wave breaking:

$$
A(z) \equiv\left(\frac{2 N}{k \bar{\rho}_{0}}\right)^{1 / 2} \frac{e^{\left(z-z_{0}\right) / 2 H}\left|F_{0}(c)\right|^{1 / 2}}{|\bar{u}-c|^{3 / 2}}=1,
$$

where $F_{0}(c)$ is the momentum flux at the source level $z_{0}$ of a wave with phase speed $c$. Above its breaking level, the wave deposits momentum in the mean flow to an extent that it remains statically stable, that is, such that $A$ does not exceed unity. The resulting drag force is (Lindzen 1981)

$$
X=-\frac{k}{2} \frac{(\bar{u}-c)^{3}}{N}\left[\frac{1}{H}-\frac{3 \bar{u}_{z}}{(\bar{u}-c)}\right] .
$$

This is often multiplied by a so-called intermittency factor, usually denoted as $\varepsilon$, which is supposed to measure the percentage of the time that the waves are actually being forced.

The drag as given by (3.2) does not depend directly on $F_{0}$, and in this sense the scheme differs from the HL72 scheme. Changing $F_{0}$, however, affects the breaking levels [according to (3.1)] and, hence, the drag profile. The magnitude of the drag depends linearly on the horizontal wavenumber $k$. However, since the choice of the intermittency factor $\varepsilon$ is quite arbitrary, one could always adjust $\varepsilon$ to compensate for changes in $k$. According to (3.1), the horizontal wavenumber also affects the breaking level of a wave and thus the choice of $k$ also affects the possible choices of $F_{0}(c)$. There is an- other obvious difference from the HL72 scheme. In the HL72 scheme, drag is always deposited at all levels, because the waves are thermally damped.

For comparison with our HL72 equatorial planetary wave simulations, we chose the same wave input parameters as in section 2 . The configuration (2.3), when used in the Lindzen scheme with $\varepsilon=1$, gives a QBOlike oscillation over the whole range of heights from the source level up to the lid of the computational domain and is used in the rest of this section. It must be noted that the choice of $k=2 \pi /\left(4 \times 10^{7} \mathrm{~m}\right)$ (wavenumber 1 ) corresponds to an unrealistically long wavelength for a gravity wave. However, changing $k, F_{0}$, and $\varepsilon$ in such a way that $F_{0}^{ \pm} / k$ remains fixed does not affect the breaking level of a wave and if $\varepsilon k$ is also kept fixed, then the magnitude of the drag is also unchanged. This means that the drag profile obtained with the configuration (2.3) and $\varepsilon=1$ could also be obtained using a shorter horizontal wavelength. The following choice of parameters, for example, would give the same drag profile as (2.3):

$$
\begin{aligned}
F_{0}^{ \pm} / \rho_{0} & = \pm 0.7 \mathrm{~m}^{2} \mathrm{~s}^{-2}, \quad c^{ \pm}= \pm 25 \mathrm{~m} \mathrm{~s}^{-1}, \\
k^{ \pm} & =2 \pi /\left(4 \times 10^{5} \mathrm{~m}\right), \quad \varepsilon=0.01 .
\end{aligned}
$$

To illustrate the mechanism for the shear zone descent, consider a configuration in which the initial mean wind takes the form of a westerly jet, as shown in Fig. 2a, and the wave phase speeds are $c^{ \pm}= \pm 25 \mathrm{~m} \mathrm{~s}^{-1}$. The solid and dashed curves in Fig. 2b show the graphs of $A^{+}$and $A^{-}$[the expression (3.1), evaluated for the westerly 
wave and the easterly wave, respectively], corresponding to the initial mean wind profile in Fig. 2a. The breaking levels $z_{b}^{ \pm}$correspond to $A^{ \pm}=1$. The expression (3.2) for the drag from the westerly wave applies above $z_{b}^{+}$and up to the level where $\bar{u}-c-3 H \bar{u}_{z}$ first becomes zero, that is, where $\partial A^{+} / \partial z=0$. This level is denoted by $z_{\max }^{+}$in Fig. 2b. Above $z_{\max }^{+}$, the drag $X^{+}$ from the westerly wave is zero, up to the level where $A^{+}(z)=A^{+}\left(z_{\max }^{+}\right)$. This is a second breaking level for the westerly wave and is denoted by $z_{b 2}^{+}$in Fig. $2 b$. So $X^{+}$is nonzero in the ranges of altitudes $z_{b}^{+}<z<z_{\max }^{+}$ and $z>z_{b 2}^{+}$. For the easterly wave, on the other hand, $A^{-}$is an increasing function of $z$ everywhere above the initial breaking level $z_{b}^{-}$, and so its drag $X^{-}$is nonzero (negative) everywhere above this level. Above $z_{b 2}^{+}$, where both $X^{+}$and $X^{-}$are nonzero and $\bar{u}$ is zero, the total drag is zero. The initial profile of the total drag due to the two waves is shown in Fig. 2c. The total drag is positive in the range $z_{b}^{+}<z<z_{\max }^{+}$, where $X^{+}$is positive and $X^{-}$is zero, and it is negative in the range $z_{b}^{-}<z<z_{b 2}^{+}$, where $X^{-}$is negative and $X^{+}$is zero. Elsewhere, it is zero.

The combined effect of the two waves is such that $\bar{u}$ eventually becomes negative at high levels (between $z_{b}^{-}$ and $z_{b 2}^{+}$) and becomes larger (more positive) in the lower levels below the jet maximum. Consequently, an easterly jet eventually forms above the westerly jet, until $A^{-}$also develops a local maximum. Once $A^{-}$develops a local maximum, an analogous situation occurs for the easterly wave to that described in the preceding paragraph for the westerly wave. The drag from the easterly wave is switched off above the level $z_{\max }^{-}$where the maximum occurs, and remains zero up to the level $z_{b 2}^{-}$where $A^{-}(z)=A^{-}\left(z_{\max }^{-}\right)$. This is a second breaking level for the easterly wave. The possibility of multiple breaking levels for each wave is an important characteristic of the Lindzen scheme (McFarlane 1987).

Without diffusion, $\bar{u} \rightarrow c^{ \pm}$in the shear zones above the lowest breaking levels $z_{b}^{ \pm}$. This has the effect of shielding the higher levels from the waves propagating up from below. According to (3.1), as $\bar{u} \rightarrow c^{ \pm}, A^{ \pm} \rightarrow \infty$. Eventually $A^{+}\left(z_{\max }^{+}\right)$and $A^{-}\left(z_{\max }^{-}\right)$become so large that there are no levels above $z_{\max }^{+}$and $z_{\max }^{-}$where either $A^{+}(z)=A^{+}\left(z_{\max }^{+}\right)$or $A^{-}(z)=A^{-}\left(z_{\max }^{-}\right)$. This means that there are no additional breaking levels above $z_{\max }^{+}$and $z_{\max }^{-}$, and so $X^{+}$and $X^{-}$become zero everywhere above $z_{\max }^{+}$and $z_{\max }^{-}$, respectively. Without any wave drag, the mean flow reaches a steady state. Vertical diffusion is needed to prevent this situation from occurring. By reducing the strength of the wind in the regions of high shear above $z_{b}^{ \pm}$, diffusion prevents $A^{ \pm}\left(z_{\max }^{ \pm}\right)$from becoming too large; consequently, the waves are able to continue depositing momentum at the higher levels. The mean flow then evolves into a state of alternating westerly and easterly regimes that move downward, becoming increasingly shallow with time, and are subsequently reduced by diffusion. With an appropriate choice of input parameters, this oscillating state can be made to resemble a QBO.

Figure 3a shows the time-height plot of the evolution of the wind when the configuration shown in Fig. 2 is used, and Figs. 3b,c show the wind and drag profiles at the end of the 12-yr model run. To obtain a realistic period ( $\sim 28$ months) with this configuration, the vertical diffusivity parameter is set to $\nu=0.4 \mathrm{~m}^{2} \mathrm{~s}^{-1}$, a larger value than that used in the HL72 simulation shown in Fig. 1. The period of the oscillation depends on the strength of the drag, which depends directly on $\varepsilon k$, and on the vertical wavelength of the oscillation, which is determined by $\nu$. Vertical diffusion affects the evolution of the mean wind in several ways. First, as noted above, increasing $\nu$ reduces $|\bar{u}|$ and hence reduces $A^{ \pm}\left(z_{\text {max }}^{ \pm}\right)$; this has the effect of lowering the upper breaking levels $z_{b 2}^{ \pm}$. The distance between subsequent breaking levels (e.g., the distance $\Delta z$ in Fig. 3c or 3f) determines the vertical wavelength of the mean-wind oscillation; thus, increasing $\nu$ gives a shorter vertical wavelength. Vertical diffusion increases the rate of downward propagation of the higher breaking levels. These effects result in the period of the oscillation being shorter and can be seen by comparing the graphs in Figs. $3 \mathrm{~d}-\mathrm{f}$, which were obtained with $\nu=0.6 \mathrm{~m}^{2} \mathrm{~s}^{-1}$, with those in Figs. 3a-c for which $\nu=0.4 \mathrm{~m}^{2} \mathrm{~s}^{-1}$.

Unlike in the HL72 scheme, it is not enough to have vertical diffusion only in a layer above the source level. To generate a QBO, diffusion is needed in the vicinity of each of the breaking levels; in practice, this means that $\nu$ must be nonzero at all levels. If the second-order diffusion is replaced, for example, by Rayleigh friction, the wind is simply damped to zero everywhere and a steady state with zero wind is attained (not shown here). This is the same situation that occurs when Rayleigh friction is included in the HL72 model and, as noted by Plumb (1977), it is due to the fact that Rayleigh friction does not allow momentum transfer across the levels where $\bar{u}$ changes direction. This observation is consistent with the findings of Dunkerton (2000); he was able to simulate the QBO by incorporating a momentum-conserving shear adjustment scheme into his model, instead of explicitly adding vertical diffusion.

Figure 3a illustrates a characteristic feature of the Lindzen gravity wave drag scheme, namely, that multiple shear zones of the same sign can coexist at different levels in a QBO driven by only two waves. This feature is a consequence of the existence of multiple breaking levels for each wave. In theory, there is no 
(a)

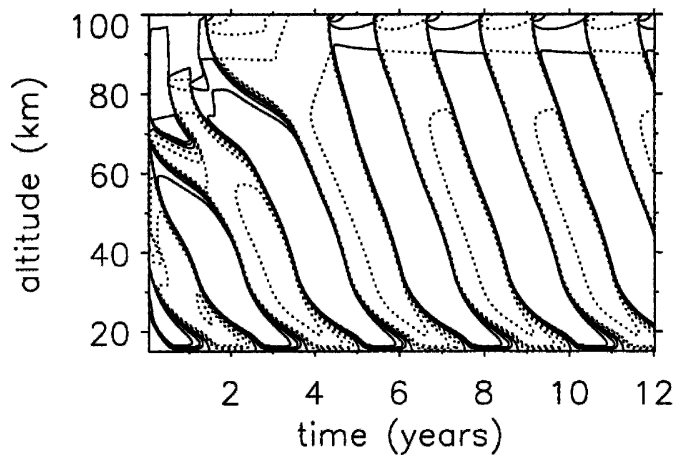

(d)

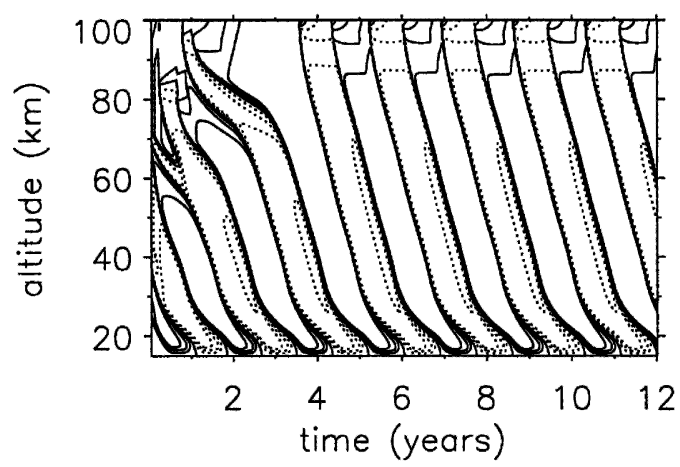

(b)

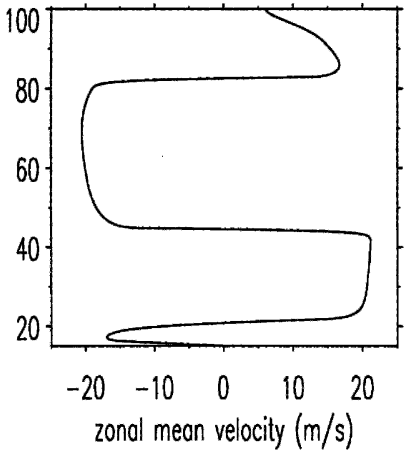

(e)

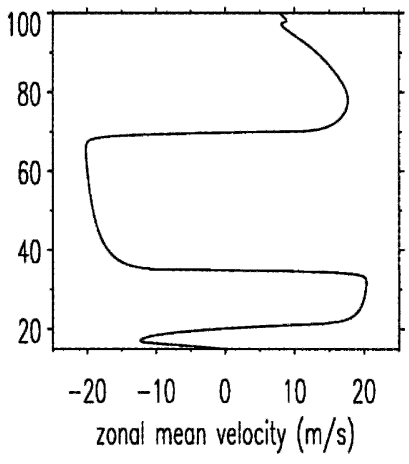

(c)

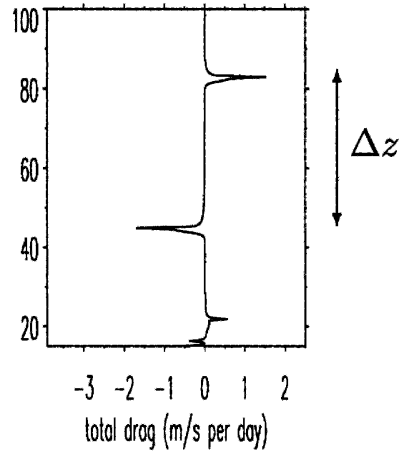

(f)

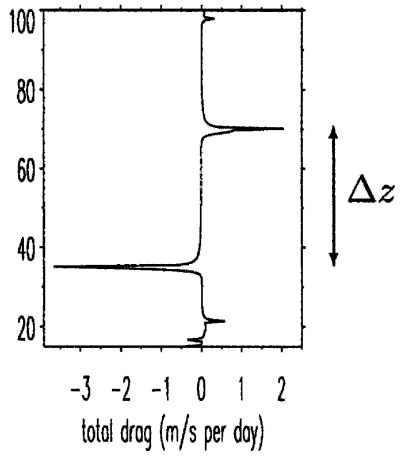

FIG. 3. Lindzen parameterization with two waves with phase speeds $c^{ \pm}= \pm 25 \mathrm{~m} \mathrm{~s}^{-1}$. (a) Time-height plot of $\bar{u}$ with $\nu=0.4 \mathrm{~m}^{2} \mathrm{~s}^{-1}$ for the initial configuration shown in Fig. 2. The solid contours denote westerlies, including the zero-wind line, and the dotted contours denote easterlies. Contour intervals are $5 \mathrm{~m} \mathrm{~s}^{-1}$. (b) Mean velocity $\bar{u}$, and (c) total drag $X$ at the end of the 12-yr run. (d) The same as for (a), but with $\nu=0.6 \mathrm{~m}^{2} \mathrm{~s}^{-1}$. (e), (f) The same as for (b), (c), but with $\nu=0.6 \mathrm{~m}^{2} \mathrm{~s}^{-1}$ and at time $t=11.5 \mathrm{yr}$ when the oscillation is in phase with that shown in (b), (c). With stronger diffusion, the vertical wavelength of the oscillation is shorter and so is the period.

limit to the number of breaking levels a wave can have, or to the number of shear zones of the same sign that can exist one above the other. Thus, the upper level of the QBO winds could, in theory, be at infinity, at least in the limit of infinitely large time.

As in the HL72 scheme, if the wave forcings are equal and opposite [i.e., $k^{-}=k^{+}, c^{-}=-c^{+}$and $F_{0}\left(c^{-}\right)$ $\left.=-F_{0}\left(c^{+}\right)\right]$, then the mean wind must be nonzero initially, otherwise the breaking levels of the waves coincide and their drag forces cancel each other out. The interesting question is whether the zero-wind initial state is unstable to small perturbations. In practice, as long as $\bar{u}$ is nonzero above the lowest breaking level of the waves, it is possible to generate a QBO. For example, if in the configuration shown in Fig. 2, the initial wind profile is replaced by one in which the maximum wind speed is $\bar{u}_{\max }=5 \mathrm{~m} \mathrm{~s}^{-1}$ instead of $20 \mathrm{~m} \mathrm{~s}^{-1}$, then $A^{+}(z)$ does not have a local maximum, that is, $\partial A^{+} / \partial z$ $>0$ everywhere, as shown in Fig. 4. This is because $\bar{u}-$ $c-3 H \bar{u}_{z}<0$ everywhere, and it implies that the drag
$X^{+}$from the westerly wave is nonzero (positive) everywhere above $z_{b}^{+}$. The ranges of altitudes affected by the two waves will then overlap initially. In the regions affected by both waves and where $\bar{u} \neq 0$, the drag is negative, since $\bar{u}$ is positive. With time, $\bar{u}$ becomes more positive in the region between $z_{b}^{+}$and $z_{b}^{-}$and eventually $A^{+}$develops a local maximum. This is the situation shown in Fig. 2 and a QBO can then result (provided there is sufficient vertical diffusion).

In general, it is possible to generate a QBO even with a small perturbation to a zero wind profile, depending on whether the following two conditions are satisfied: (i) The wave amplitudes and the initial wind must be such that either the waves do not break at the same level (as in Fig. 4) or, if they do break at the same level, then the wind must be nonzero above the breaking level, so that the total drag from the two waves above their breaking level is also nonzero. (ii) An appropriate value of $\nu$ is chosen; if $\bar{u}$ is weak initially and $\nu$ is large, the wind may be suppressed to the extent that it may be 
(a)

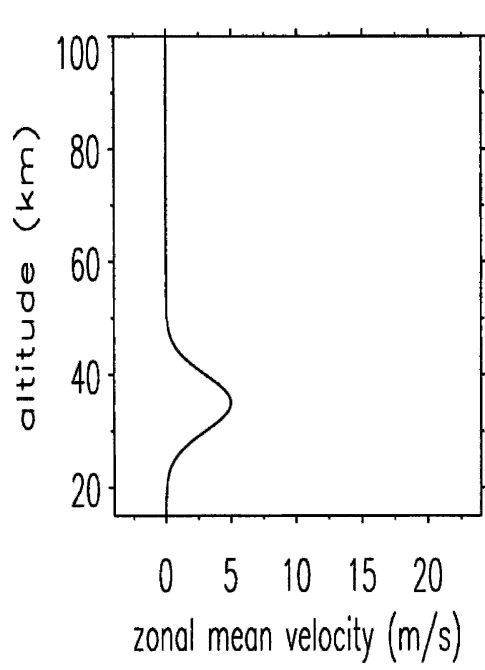

(b)

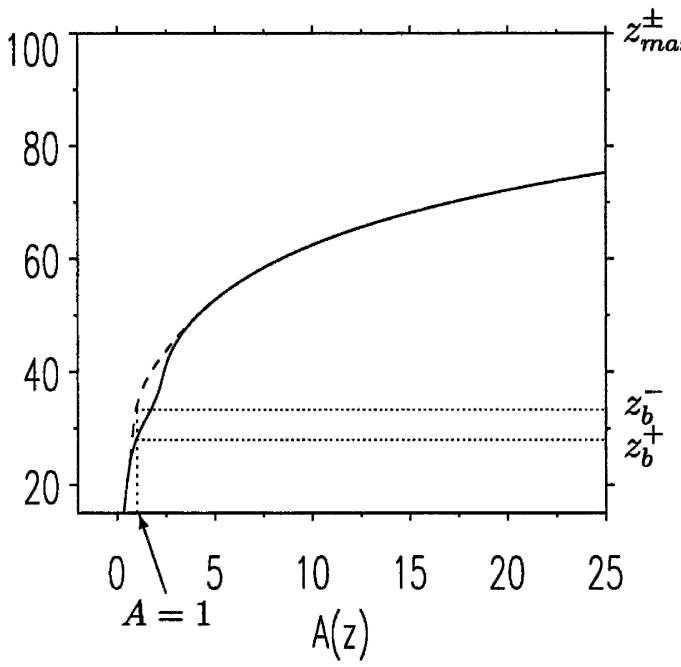

(c)

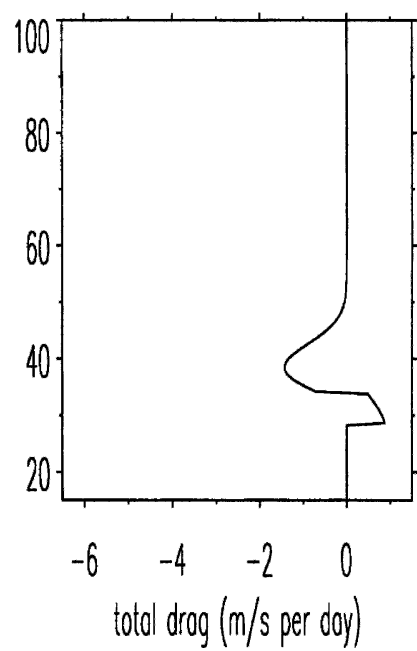

FIG. 4. Lindzen parameterization with two waves with phase speeds $c^{ \pm}= \pm 25 \mathrm{~m} \mathrm{~s}^{-1}$. (a) Initial mean velocity with maximum value of $\bar{u}_{\max }=5 \mathrm{~m} \mathrm{~s}^{-1}$, (b) the function $A^{ \pm}(z)$ defined in (3.1), and (c) the total drag from the two waves.

impossible to reach an oscillating state. For example, in the configuration considered here, with $\bar{u}_{\max }$ as small as $0.01 \mathrm{~m} \mathrm{~s}^{-1}$, it is possible to generate a QBO only if $\nu$ is smaller than about $0.3 \mathrm{~m}^{2} \mathrm{~s}^{-1}$. It must be noted, however, that a situation with such weak shear is of theoretical interest only, since in reality such weak winds are unlikely to occur in a more realistic model that includes wave driving from resolved planetary-scale equatorial waves as well as gravity waves. Wind variations would typically be of the order of those shown in Figs. 2 and 4 or larger, in which case a choice of $\nu$ in the range $0.3-0.6 \mathrm{~m}^{2} \mathrm{~s}^{-1}$ would be satisfactory.

\section{Alexander and Dunkerton's parameterization}

The parameterization described by Alexander and Dunkerton (1999, hereafter AD99) is a variant of the Lindzen scheme in which each wave is assumed to deposit all its momentum at its breaking level. A continuous spectrum of waves over a range of phase speeds is assumed, in order to have a drag profile that is a continuous function of height, and in this sense, the scheme is similar to, and may be considered as an extension of, the continuous spectrum scheme of Lindzen and Holton (1968). In practice, however, the spectrum is discretized into a finite number of waves. An additional component of the AD99 scheme is that it takes into account the nonhydrostatic effect of total internal reflection of the waves. The waves that are reflected are those with large intrinsic phase speeds. Waves with small intrinsic phase speeds, on the other hand, break at low levels and are not reflected.

To implement the scheme, one starts at the source level and removes from the spectrum any waves that would have already reflected or broken and then works upward, at each level, testing each of the remaining waves to determine whether the wave would break or be reflected in an interval (of height $\Delta z$, say) centered at that level. Reflected waves are removed from the spectrum at that level and all subsequent levels; breaking waves deposit their momentum at that level. The drag is calculated by summing over the waves that break in the height interval $\Delta z$ and multiplying by an intermittency factor $\varepsilon$. In AD99's implementation of the scheme, the total time average momentum flux in the spectrum is specified and $\varepsilon$ is chosen to be proportional to the ratio of this quantity to the sum of the discrete momentum fluxes $F_{0}(c)$ over the spectrum [see (19) in AD99]. In this way, $\varepsilon$ is proportional to the phase speed resolution, that is, $\varepsilon \propto 1 / N_{c}$, where $N_{c}$ is the number of waves in the spectrum, and so changing the phase speed resolution does not affect the magnitude of the calculated drag. In the present study, the effect of reflection is neglected and we also assume that all the waves in the spectrum have the same horizontal wavenumber.

In our simulations of the QBO using the AD99 scheme, we observe that, as in the Lindzen scheme, the period of the zonal wind oscillation is controlled by the horizontal wavenumber $k$, the intermittency factor $\varepsilon$ and the vertical diffusivity $\nu$. Unlike in the Lindzen scheme, however, the ability of the scheme to generate a QBO depends on the specification of the initial wind profile. To illustrate this, it is helpful to examine the special case where the momentum flux at the source 
(a)

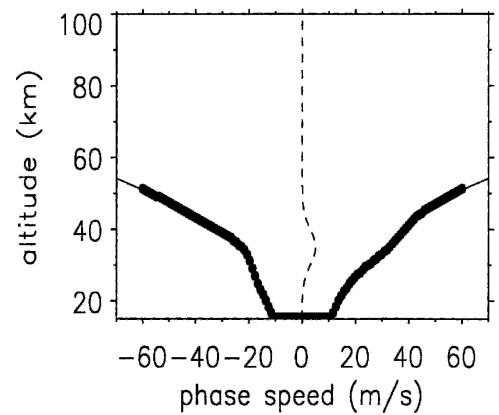

(b)

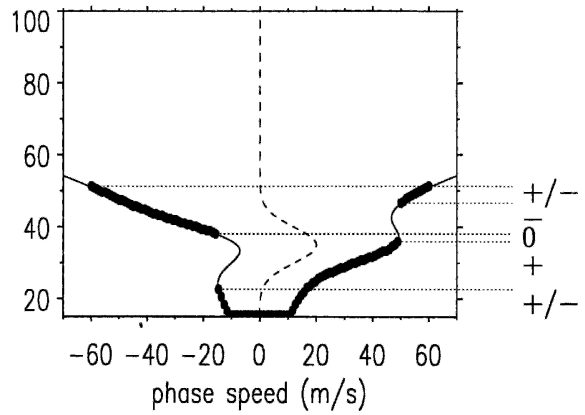

FIG. 5. AD99 parameterization with the momentum flux spectrum $F_{0}(c)=(\operatorname{sgn} c) \times$ a constant. (a) The dotted line shows the mean velocity with maximum value of $\bar{u}_{\max }=5 \mathrm{~m} \mathrm{~s}^{-1}$. The thin solid line shows the graph of $c^{ \pm}\left(z_{b}\right)=\bar{u}\left(z_{b}\right) \pm \alpha e^{z_{b} / 3 H}$ and the black dots show the actual breaking levels of the waves. (b) The same as for (a), but with stronger shear: $\bar{u}_{\max }=$ $20 \mathrm{~m} \mathrm{~s}^{-1}$. The profile of breaking levels is now a discontinuous function of $c$. The sign of the drag over each range of heights is shown at the right of the plot in (b).

takes the form $F_{0}(c)=(\operatorname{sgn} c) \times$ a constant. For each $c$, the breaking level $z_{b}(c)$ is determined by Lindzen's criterion,

$$
\frac{\alpha e^{z_{b} / 3 H}}{\left|\bar{u}\left(z_{b}\right)-c\right|}=1
$$

where

$$
\alpha=\left|\frac{2 N}{k \bar{\rho}_{0}} e^{-z_{0} / H} F_{0}(c)\right|^{1 / 3},
$$

and with the source spectrum $F_{0}(c)=(\operatorname{sgn} c) \times$ a constant, $\alpha$ is independent of $c$. This means that $c$ can be written as an explicit function of $z_{b}$,

$$
c^{ \pm}\left(z_{b}\right)=\bar{u}\left(z_{b}\right) \pm \alpha e^{z_{b} / 3 H} .
$$

Depending on the initial strength of the shear, there are then two possibilities. These are illustrated in Fig. 5 for an initial $\bar{u}$ profile in the form of a westerly jet with maximum amplitude at $z=35 \mathrm{~km}$. In Fig. 5a, $\bar{u}=5$ $\mathrm{m} \mathrm{s}^{-1}$ at its maximum; this is the same wind profile used in Fig. 4. In Fig. $5 b, \bar{u}=20 \mathrm{~m} \mathrm{~s}^{-1}$ at its maximum and the wind profile is the same as that in Fig. 2. In both cases, $k^{ \pm}=2 \pi /\left(4 \times 10^{7} \mathrm{~m}\right)$ and

$$
\begin{aligned}
& F_{0}(c) / \bar{\rho}_{0} \\
& =\left\{\begin{array}{cll}
(\operatorname{sgn} c) \times 5 \times 10^{-3} \mathrm{~m}^{2} \mathrm{~s}^{-2} & \text { for } & |c| \leq 60 \mathrm{~m} \mathrm{~s}^{-1} \\
0 & \text { for } & |c|>60 \mathrm{~m} \mathrm{~s}^{-1}
\end{array}\right.
\end{aligned}
$$

\section{a. Case 1: Weak shear}

If the initial shear is so weak that there is no level at which $\partial c^{ \pm} / \partial z_{b}$ changes sign (i.e., if $c^{+}$is an increasing function and $c^{-}$is a decreasing function of $z_{b}$ ), then, at every level $z$, both easterly and westerly waves contribute to the drag. The two components of the drag are given by

$$
X^{ \pm}=\varepsilon \frac{\left|F_{0}\left[c^{ \pm}(z)\right]\right|}{\Delta c \bar{\rho}(z)} \frac{\partial c^{ \pm}}{\partial z},
$$

where $\Delta c$ is the phase speed resolution. [This expression was derived by Dunkerton (1997) and AD99.] Thus, from (4.3), the total drag at height $z$ is

$$
X=2 \varepsilon \frac{\left|F_{0}\left[c^{ \pm}(z)\right]\right|}{\Delta c \bar{\rho}(z)} \frac{\partial \bar{u}}{\partial z},
$$

and the drag increases exponentially with height and also depends on the amplitude of the waves at the source. In the absence of diffusion, the equation for the time evolution of $\bar{u}$ then takes the form

$$
\frac{\partial \bar{u}}{\partial t}+f(z) \frac{\partial \bar{u}}{\partial z}=0
$$

with

$$
f(z)=-\frac{2 \varepsilon\left|F_{0}\right|}{\Delta c \bar{\rho}_{0}} e^{\left(z-z_{0}\right) / H},
$$

where $\bar{\rho}_{0}$ is the background density at the source level $z_{0}$. The solution to (4.7) can be found quite readily by the method of characteristics to be

$$
\bar{u}(z, t)=\bar{u}_{\text {initial }}(\tau)
$$

where $\tau$ is given by

$$
e^{-\tau / H}=e^{-z / H}-\frac{2 \varepsilon\left|F_{0}\right|}{H \Delta c \bar{\rho}_{0}} e^{-z 0 / H} t .
$$


(a)

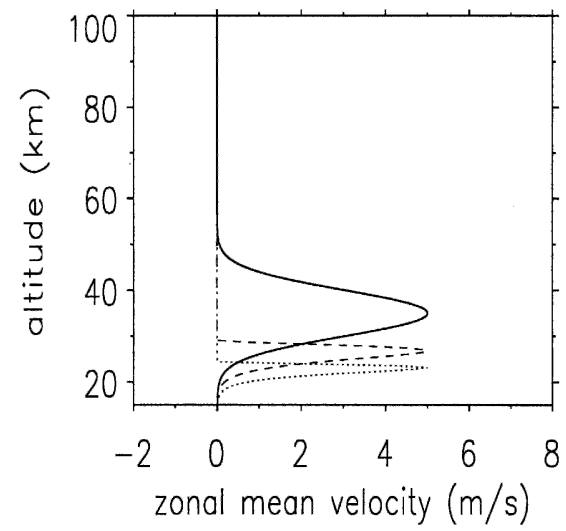

(b)

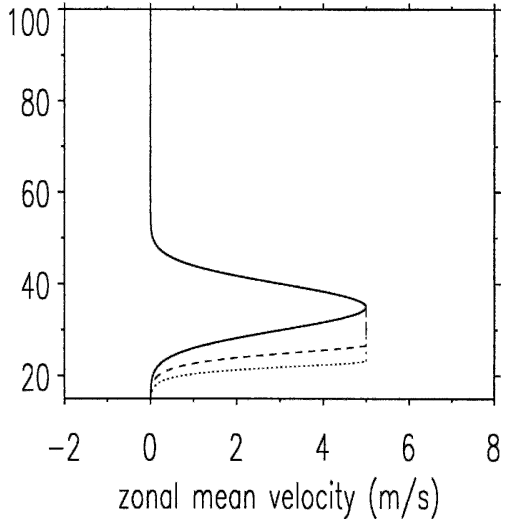

FIG. 6. (a) AD99 parameterization with the momentum flux spectrum $F_{0}(c)=(\operatorname{sgn} c) \times$ a constant and the initial mean velocity profile given by the dashed line in Fig. 5a. The evolution of $\bar{u}$ with time in the absence of diffusion, according to the exact solution, is shown by the solid, dashed, and dotted lines, corresponding to $t=0,2000$, and 4000 days, respectively. (b) Same configuration as (a), but using the LH68 parameterization instead.

This solution describes a jet moving downward with no change in amplitude, as shown in Fig. 6a. The speed $f(z)$ of downward propagation of the jet increases exponentially with height; so the downward propagation is faster at higher levels. The speed of descent of the jet also depends on the amplitude of the waves at the source level. Clearly, if the evolution of $\bar{u}$ were to continue indefinitely as shown in Fig. 6a, an oscillating state would never be attained, since $\bar{u}$ would remain nonnegative everywhere for all time. However, the mean flow evolution shown in Fig. 6a does not, in general, continue indefinitely. With time, as the jet moves downward and becomes increasingly narrow, $|\partial \bar{u} / \partial z|$ increases at the lower levels and can eventually become large enough that

$$
\frac{\partial \bar{u}}{\partial z}=\mp \frac{\alpha}{3 H} e^{z / 3 H}
$$

and hence $\partial c^{ \pm} / \partial z=0$, at some level. This is the situation defined as case 2 and discussed in detail below. In this situation, the range of levels affected by the westerly waves does not coincide exactly with the range affected by the easterly waves and, at different levels, the drag can be positive, negative, or zero. This allows the formation of easterly shear zones even if none existed initially, and hence allows the development of a $\mathrm{QBO}$, as described in our discussion of case 2. In practice, noise from the numerical evaluation of the wave drag could also create local maxima/minima in the profiles of $c^{ \pm}(z)$, and thus allow the formation of easterly shear zones and a QBO.

An important factor that could affect the develop- ment of a QBO under these circumstances is the inclusion of vertical diffusion. If the vertical diffusion term is restored to (4.7), the jet still moves downward, but its amplitude decreases with time. With too-strong diffusion, $\bar{u}$ could be damped to zero too fast, thus preventing the system from ever reaching a state where $\partial c^{ \pm} / \partial z$ could be zero somewhere. Instead, it would tend toward a steady state with zero wind.

It is interesting to note that in case 1, the drag profile and the evolution of the mean wind are similar to those obtained by Lindzen and Holton (1968, hereafter LH68). In their model, there is a continuous spectrum of upward-propagating waves (over a range of phase speeds) and each wave is completely absorbed at its critical level, that is, the level where $\bar{u}(z)=c$. Booker and Bretherton (1967) showed that linear absorption of a wave at its critical level causes the momentum flux of the wave to be reduced across the critical level by a factor of $\exp \left[-2 \pi\left(\mathrm{Ri}_{c}-1 / 4\right)\right]$, where $\mathrm{Ri}_{c}$ is the local Richardson number at the critical level. Based on this result, LH68 obtained an approximate expression for the drag due to the wave absorption. AD99 pointed out that LH68's expression can be written as

$$
X=\varepsilon \frac{\left|F_{0}(\bar{u})\right|}{\Delta c \bar{\rho}(z)} \frac{\partial \bar{u}}{\partial z},
$$

which is of the same form as (4.6). Since each wave is completely absorbed at its critical level, the total drag must be zero above any levels where $\partial \bar{u} / \partial z$ changes sign. For example, with the jet profile shown in Fig. 6, since the wind initially has a maximum at $z=35 \mathrm{~km}$, the region above this level is shielded from the waves 
propagating up from below. Consequently, the drag remains zero above this level for all time and only the lower half of the jet propagates downward. This is seen in Fig. 6b, which shows the results of a simulation using the LH68 parameterization with the same input parameters as in Fig. 6a. Figure 6b can be contrasted with Fig. 6a and illustrates the difference between the LH68 and the AD99 schemes. It is impossible to get a spontaneous QBO-like oscillation with the wind profile in Fig. $6 \mathrm{~b}$. The shear zones propagate downward, but the wind cannot change direction. LH68 got around this constraint by applying an oscillating upper boundary condition around the level of the stratopause to mimic the semiannual oscillation.

With the drag in the LH68 scheme written in the form (4.12), the relationship between the LH68 and AD99 scheme becomes apparent. In the limit as the wave amplitude goes to zero in (4.3), $\alpha \rightarrow 0$, and so $c^{ \pm} \rightarrow \bar{u}$. In that limit, the AD99 drag defined by (4.6) takes the form (4.12). Thus, the LH68 representation of the drag corresponds to the small-amplitude limit of AD99's representation, and Fig. 6b may be interpreted as such.

Equation (4.7) illustrates an important characteristic of the AD99 scheme, namely, that there is downward propagation of information, even in the absence of vertical diffusion. This is a characteristic feature of the LH68 scheme as well, and has also been observed in other situations, such as in the transient wave-meanflow interaction studied by Dunkerton (1981a,b, 1982). An additional characteristic of the weak-shear configuration of the AD99 scheme, which is also true of the LH68 scheme, is that the evolution of the wind at a given level depends only on the local shear and is independent of the flow evolution at lower levels. This is in contrast to the HL72 scheme for thermally damped equatorial planetary waves, in which there is downward propagation of phase only and the wind evolution is driven by the wind at lower levels (Plumb 1977).

\section{b. Case 2: Strong shear}

With sufficiently strong shear, $\partial c^{ \pm} / \partial z_{b}$ changes sign at one or more places, as shown in Fig. 5b. Thus, the range of levels affected by the westerly waves does not coincide exactly with the range affected by the easterly waves, although they could overlap. There are four kinds of regions: the overlapping regions in which the total drag is given by the expression (4.6), the regions affected by only westerly waves and by only easterly waves, where the drag is given by (4.5) with the plus sign and with the minus sign respectively, and the regions where the drag is zero. These regions are indicated in Fig. $5 b$ by the labels $(+/-),(+),(-)$, and $(0)$ respectively. In the $(+/-)$ region, a westerly jet propagates downward with speed $f(z)$ given by (4.8), and remains positive, as in (4.9). In the $(+)$ and $(-)$ regions, (4.5) implies that the equation for $\bar{u}$ is of the form

$$
\frac{\partial \bar{u}}{\partial t}+\frac{f(z)}{2} \frac{\partial \bar{u}}{\partial z}= \pm h(z),
$$

where

$$
h(z)=\frac{\varepsilon\left|F_{0}\right|}{\Delta c \bar{\rho}(z)} \frac{\alpha}{3 H} e^{z / 3 H} .
$$

The plus sign on the right-hand side of (4.13) applies in the $(+)$ region, and the minus sign in the $(-)$ region. Thus, the jet moves downward, but with half the speed as in the $(+/-)$ regions. The nonzero term on the righthand side of (4.13) means that $\bar{u}$ is of the form

$$
\bar{u}(z, t)=\bar{u}_{\text {initial }}(\tau) \pm \int_{0}^{t} h[z(t, \tau)] d t
$$

where $z, t$, and $\tau$ are now related by

$$
e^{-\tau / H}=e^{-z / H}-\frac{\varepsilon\left|F_{0}\right|}{H \Delta c \bar{\rho}_{0}} e^{-z_{0} / H} t .
$$

Since the function $h$ is always positive, (4.15) implies that in the $(+)$ region, $\bar{u}$ becomes more positive, and in the $(-)$ region, $\bar{u}$ becomes more negative. Thus, $\bar{u}$ eventually becomes negative in the $(-)$ region and an oscillation in $\bar{u}$ would then be possible. The key difference between this configuration and that in case 1 is that the net drag at any level now depends on the evolution of the wind at lower levels as well as on the local shear. To generate a $\mathrm{QBO}$, the total drag corresponding to a given value of $\bar{u}$ must take both positive and negative values over each oscillation period. In case 1 , the drag is a single-valued function of the local shear and thus an oscillation between positive and negative values is impossible. In case 2 , because of the multivalued relation between $c^{ \pm}$and $z$, whether the drag from the wave with phase speed $c^{ \pm}$is deposited at $z$ depends on whether it has already been deposited below and, hence, on the wind below. It is evident from Fig. 5b that any changes in the wind would affect the levels at which the drag would switch from one type of region to another.

Another point to note is that, in case 2, the AD99 configuration with $F_{0}(c)=(\operatorname{sgn} c) \times$ a constant is similar to the two-wave Lindzen scheme in the sense that there are four kinds of regions: those affected by waves with positive phase speed only, by waves with negative phase speed only, by both, or by none. We note that the breaking criterion (3.1) for the two-wave Lindzen scheme is equivalent to 
(a)

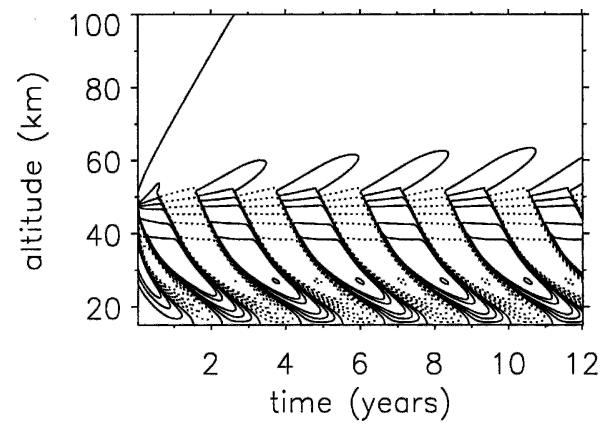

(b)

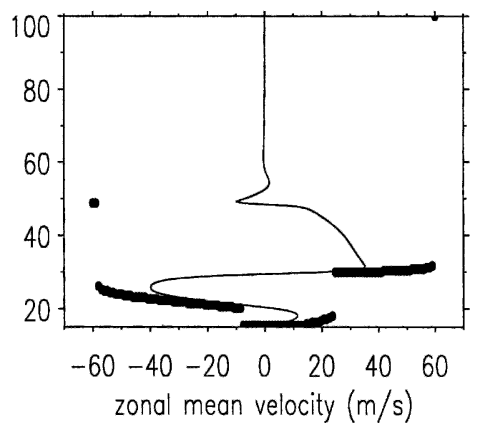

FIG. 7. (a) Time-height plot of the zonal mean wind in a QBO simulation using the AD99 parameterization with the spectrum $F_{0}(c)=(\operatorname{sgn} c) \times$ a constant and the initial mean velocity profile given by the dashed line in Fig. 5b. The solid contours denote westerlies, including the zero-wind line, and the dotted contours denote easterlies. Contour intervals are $5 \mathrm{~m} \mathrm{~s}^{-1}$. (b) The mean wind at the end of the 12-yr run. The black dots show the breaking levels of the waves.

$$
B^{ \pm}(z) \equiv \bar{u} \pm \alpha e^{z / 3 H}=c^{ \pm} .
$$

For each wave with phase speed $c=c^{ \pm}$, the breaking level $z_{b}^{ \pm}$is the lowest level at which $B^{ \pm}(z)=c^{ \pm}$. Above this level, the drag is nonzero up to the lowest level $z_{\text {max }}^{ \pm}$ where $\partial A^{ \pm} / \partial z=0$. In terms of the function $B^{ \pm}(z), z_{\max }^{ \pm}$ is the level where

$$
\frac{\partial B^{ \pm}}{\partial z}=\frac{\left(B^{ \pm}(z)-c^{ \pm}\right)}{3 H} .
$$

Thus, the breaking levels in the Lindzen scheme could be determined by examining $B^{ \pm}(z)$ instead of $A^{ \pm}(z)$.

Now $B^{ \pm}(z)$ is the same as the expression (4.3) for $c^{ \pm}(z)$ in the AD99 scheme. In that scheme, the total drag may be nonzero all the way down to the source level. That is the case for the configuration used here, since the waves with small phase speed have nonzero amplitudes and the wind is nonzero at the low levels directly above the source level. Above $z_{1}$, the lowest level where $\partial B^{-} / \partial z=0$, the drag from the easterly waves must be zero. The drag from the westerly waves also becomes zero at $z_{2}$, the lowest level where $\partial B^{+} / \partial z$ $=0$. The total drag is zero from this level up to $z_{3}$, where the drag from the easterly waves becomes nonzero again. Above the level $z_{4}$, the drag from the westerly waves also becomes nonzero, but as $\bar{u}$ is close to zero in this region, the total drag is approximately zero. In terms of the resulting drag profiles, the levels $z_{2}, z_{3}$, and $z_{4}$ are analogous to $z_{\max }^{+}, z_{b}^{-}$, and $z_{b 2}^{+}$in the twowave Lindzen scheme (Fig. 2), although their physical interpretation is different and they do not actually coincide. Also, the levels $z_{1}$ and $z_{b}^{+}$could be made to coincide if the source spectrum used in the AD99 simu- lation were to be truncated at some minimum phase speed, that is, if we set $F_{0}(c)=0$ for $|c|<c_{\min }=B^{+}\left(z_{b}\right)$.

In Fig. 7, the time-height contour plot of $\bar{u}$ is shown from a simulation in which the drag is calculated using AD99's discrete representation [given by their expression (15)]. The result shown corresponds to the initial state given by the dashed line in Fig. 5b. As predicted from the discussion of the exact solution, a QBO is obtained when this initial configuration is used. The range of QBO heights is from the source level up to a height of about $52 \mathrm{~km}$. This is because, with the truncated spectrum (4.4) used in this simulation, all the waves break below this level (as seen in Fig. 5b) and so there is no drag above. It is important to note, however, that with a sufficiently broad spectrum of waves, it is theoretically possible to obtain multiple shear zones of the same sign at different altitudes, as in the two-wave Lindzen simulations. The curvature of the wind profile in Fig. 7 is due to the fact that the drag, and hence the rate of descent of the shear zones, increases exponentially with height.

As in case 1 , vertical diffusion acts to reduce $|\bar{u}|$ in regions with large shear. With excessively strong diffusion, $|\bar{u}|$ could be reduced to the extent that the situation in case 1 would result. It would then be impossible to obtain an oscillation between positive and negative values of $\bar{u}$.

In general, the inclusion of vertical diffusion is crucial to the development of a QBO, and diffusion plays a similar role to that in the Lindzen scheme. To illustrate this, an experiment was carried out with the same configuration as in Fig. 5b, but with $\nu$ set to zero. Within 1 yr of the model run, a steady state was attained. The time-height plot of the zonal wind velocity is shown in 
(a)

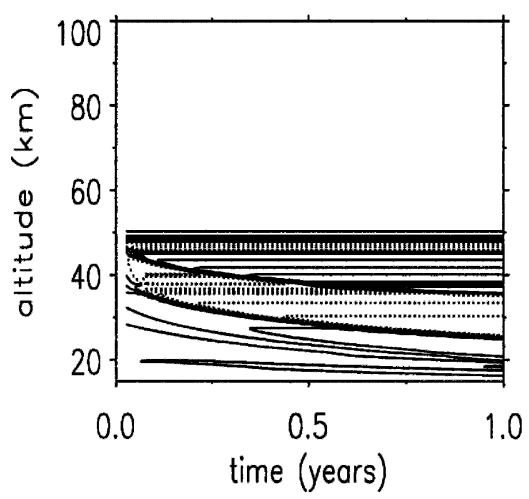

(b)

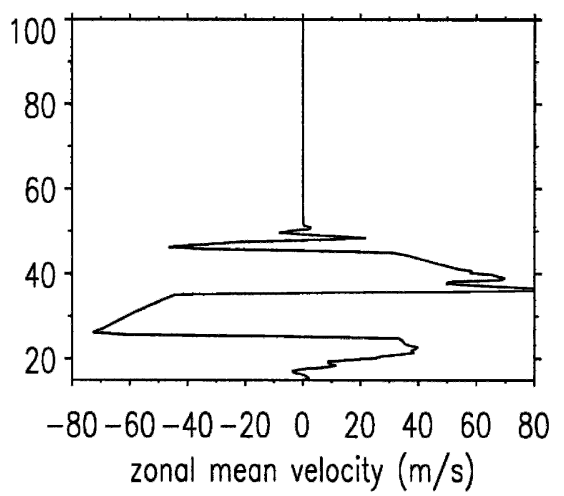

(c)

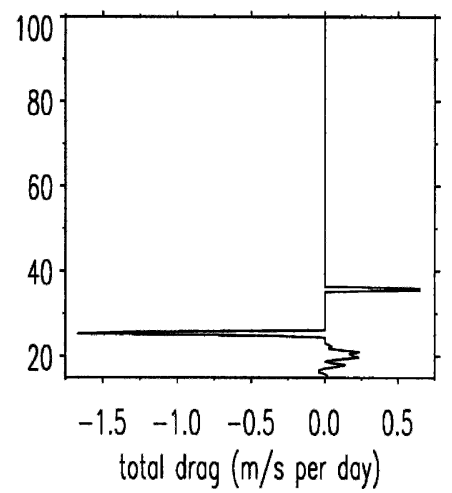

FIG. 8. (a) Time-height plot of the zonal mean wind in a QBO simulation using the AD99 parameterization with zero vertical diffusion. The initial configuration and the source spectrum is the same as in Fig. 7. The solid contours denote westerlies, including the zero-wind line, and the dotted contours denote easterlies. Contour intervals are $5 \mathrm{~m} \mathrm{~s}^{-1}$. (b) The mean wind at the end of the 2-yr run. (c) The total drag as a function of height at the end of the 2-yr run.

Fig. 8a, and the wind and the breaking levels at the end of a 2-yr model run and the corresponding drag profile are shown in Figs. 8b,c respectively. The strong shear regions that have developed at low levels prevent deposition of momentum at higher levels and, consequently, the drag is zero above a height of about $25 \mathrm{~km}$. As in the Lindzen scheme, adding diffusion at low levels (and setting the diffusion to zero at higher levels) does not solve the problem. Several experiments were carried out to verify this (not shown here). It was seen that regions of large shear eventually develop at the lowest levels of the region in which the diffusion is set to zero and these prevent momentum deposition at higher levels in the same way as in Figs. 8b,c. It follows that diffusion is needed at all levels.

Further insight into the evolution of the QBO in case 2 and into the role played by diffusion can be obtained by examining the evolution of the drag at a fixed level as a function of the wind speed or wind shear at that level. In Fig. 9a, the total drag $X$ at $z=35 \mathrm{~km}$ is plotted over the 12-yr simulation period shown in Fig. 7a as a function of the wind speed at that level. At $t=0$, the jet maximum $\bar{u}=20$ occurs at this level. As shown in Fig. $5 \mathrm{~b}$ the wave that breaks at this level is westerly and so the drag is positive (the black dot). As $t$ increases, $X$ becomes negative, eventually increases back to zero, and then stays at zero for a period of time. The process is then repeated but with opposite sign, and continues through a regular cycle thereafter. Figure $9 \mathrm{~b}$ shows the evolution of the total zonal mean acceleration $\bar{u}_{t}$ as a function of $\bar{u}$. This is equal to the wave $\operatorname{drag}(X)$, plus the forcing due to diffusion $\left(\nu \bar{u}_{z z}\right)$. During the time intervals in which the drag is zero, $\bar{u}$ is driven by the diffusion; this can be seen by comparing Figs. 9a and 9b. There are two branches of the curve in Fig. 9a corresponding to the time intervals in which $X=0$. In the left branch, $\bar{u}$ is negative initially, but $\nu \bar{u}_{z z}$ is positive and its effect is to drive $\bar{u}$ back toward zero and then to positive values. In the right branch, $\nu \bar{u}_{z z}$ is negative and so $\bar{u}$ becomes more negative with time. These time intervals are short (on the order of a few weeks), compared with the time intervals in which the drag is nonzero, and correspond to the regimes in Fig. 7 where $\bar{u}$ changes sign and both the positive and negative contours lie close to the zero-wind line.

Figure $9 \mathrm{c}$ shows the wave $\operatorname{drag} X$ as a function of $\bar{u}$, as in Fig. 9a, but for the case of zero diffusion $(\nu=0)$. The curve starts at the same place as in Fig. 9a, decreases through negative values and increases back to zero. But once $X$ reaches zero, the system comes to a standstill because there is no wave drag or diffusion to drive the mean flow.

Figure $9 \mathrm{~d}$ shows the wave $\operatorname{drag} X$ as a function of the shear $\bar{u}_{z}$ at the level $z=35 \mathrm{~km}$ and illustrates the functional relation between $X$ and $\bar{u}_{z}$. At any level, the drag can be zero, equal to $-(1 / 2) f(z) \bar{u}_{z} \pm h(z)$, or equal to $-f(z) \bar{u}_{z}$, depending on whether there are no waves breaking at that level, a single breaking wave, or breaking waves of both signs. Figure 9d shows that, at the level $z=35 \mathrm{~km}$, the third situation never actually occurs. There are time regimes in which there are no breaking waves at this level and the drag remains zero while the magnitude of the shear increases. At the end of each such regime, there is a sharp transition to a time regime in which the drag is nonzero and of the same sign as the shear, the magnitude of the shear decreases 
(a)

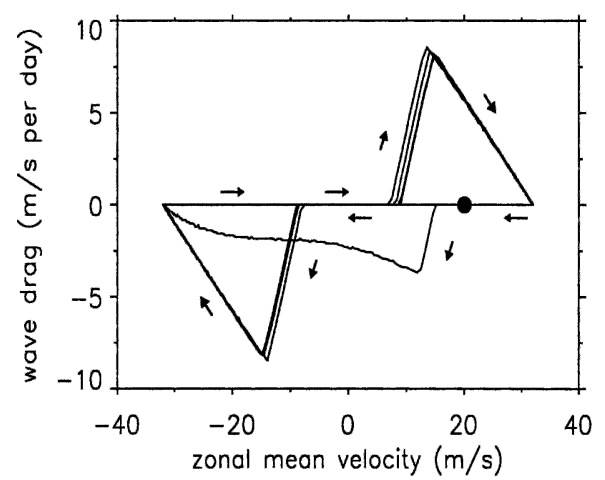

(c)

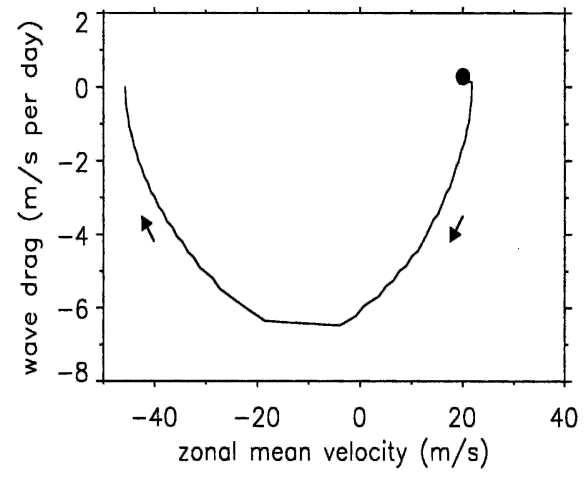

(b)

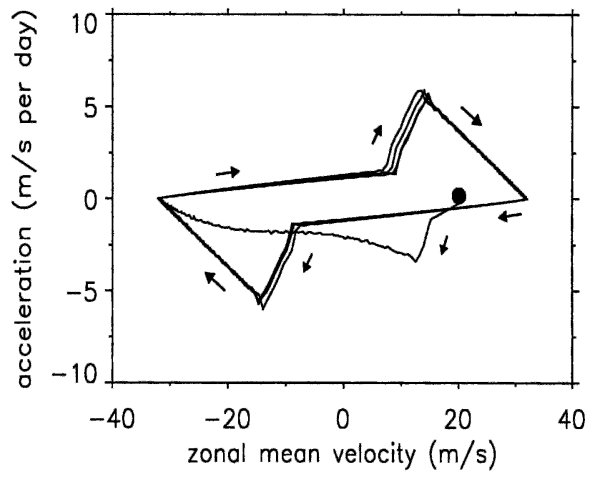

(d)

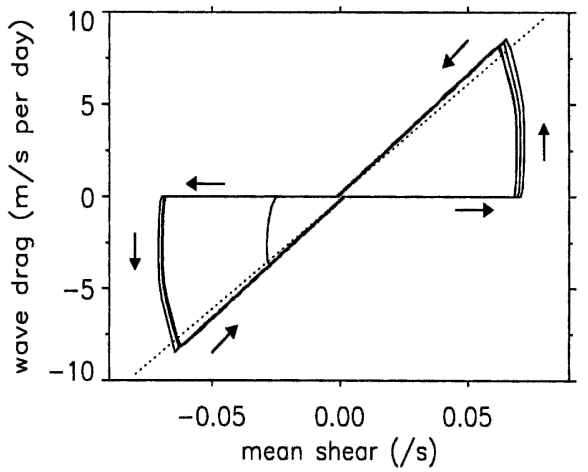

FIG. 9. (a) Here, $X$ is a function of $\bar{u}$ at $z=35 \mathrm{~km}$, (b) $X+\nu \bar{u}_{z z}$ is a function of $\bar{u}$ at $z=35 \mathrm{~km}$, and (c) $X$ is a function of $\bar{u}$ at $z=35 \mathrm{~km}$ in the case where $\nu=0$ (no diffusion). In (a)-(c), the black dot corresponds to time $t=0$. (d) Here, $X$ is a function of $\bar{u}_{z}$ at $z=35 \mathrm{~km}$. The dotted lines correspond to $-(1 / 2) f(z) \bar{u}_{z} \pm h(z)$, where $f(z)$ and $h(z)$ are given by (4.8) and (4.14), respectively. In each graph, the simulation is for a period of $12 \mathrm{yr}$ and the arrows show the direction of increase of time.

to zero and the magnitude of the drag decreases linearly with that of the shear. The branches of the curve in Fig. 9d that correspond to these latter time regimes have slopes of $-f(z) / 2$ and intersect the wave drag axis at $X= \pm h(z)$. To illustrate this, lines with slopes of $-f(z) / 2$ and intercepts of $\pm h(z) \approx \pm 0.157 \mathrm{~m} \mathrm{~s}^{-1}$ day $^{-1}$ have been added to the curve in Fig. 9d. Clearly, if (4.6) held for all time at all levels, as in case 1, it would be impossible to get a QBO because the relation between the drag and the shear would be single-signed. In that case, the curve describing the relation between the drag and shear would consist of a single straight line with slope $-f(z)$.

In summary, the ability of the AD99 scheme to generate a QBO-like oscillation depends on the balance between the strength of the vertical diffusion and the initial strength of the shear. Our numerical simulations indicate that this is true in general, that is, for other
$F_{0}(c)$ profiles, even those for which there is no explicit relationship between $c$ and $z_{b}$.

The simulations described above were repeated using the input spectrum

$$
F_{0}(c) / \bar{\rho}_{0}=(\operatorname{sgn} c) \times B_{w} e^{-\left(c / c_{w}\right)^{2}},
$$

which is of the form suggested by Alexander and Dunkerton (1999) and is shown in Fig. 10a. We set $B_{w}$ $=10^{-2} \mathrm{~m}^{2} \mathrm{~s}^{-2}$ and $c_{w}=60 / \sqrt{\pi} \mathrm{m} \mathrm{s}^{-1} \approx 33.85 \mathrm{~m} \mathrm{~s}^{-1}$, so that the total input flux of each sign, that is, the integral of $F_{0}(c)$ over the range of $c$ values of each sign, is the same as with the configuration (4.4). Waves with large phase speeds have small amplitudes and thus high breaking levels, as shown in Fig. 10b, so there is more drag at high altitudes than there is with the spectrum (4.4). This is seen in Fig. 11. The range of QBO altitudes extends up to the top of the computational domain and the maximum QBO winds occur between 60 
(a)

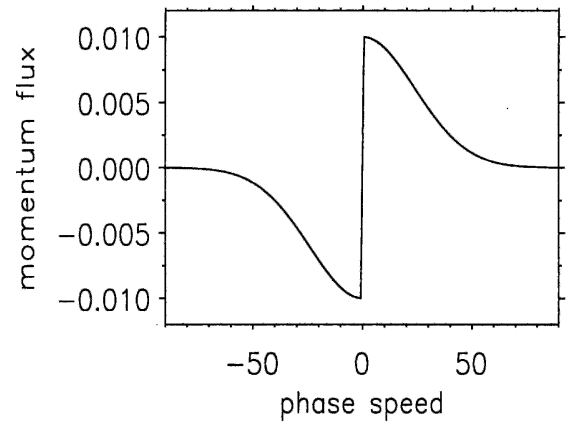

(b)

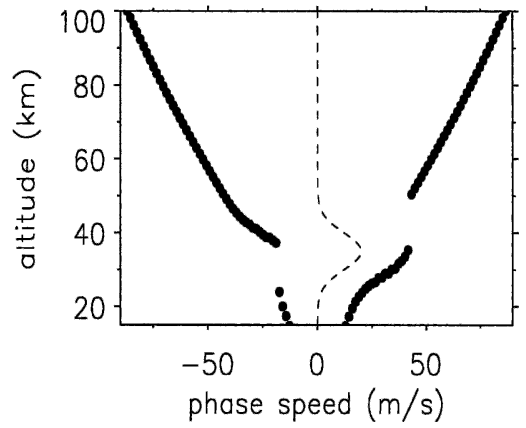

FIG. 10. (a) Momentum flux spectrum similar to that used by AD99. (b) Initial mean velocity with maximum value of $\bar{u}_{\max }=20 \mathrm{~m} \mathrm{~s}^{-1}$ (dashed line). The black dots show the initial breaking levels of the waves.

and $80 \mathrm{~km}$. As in the Lindzen scheme, there are multiple shear zones of the same sign one above the other. However, in an infinitely high domain, the wave drag would tend to zero as $z \rightarrow \infty$, since the uppermost level of the QBO winds is determined by the width of the source spectrum.

With an antisymmetric spectrum such as (4.4) or (4.19), $\bar{u}$ must be nonzero initially for the total drag to be nonzero. As in the Lindzen scheme, a small perturbation to a zero initial wind may be unstable and grow with time; in that case, a zonal wind oscillation eventually results if the choice of input parameters allows one. With $\bar{u}=0$ initially, a small perturbation to an antisymmetric spectrum may also be unstable and can lead to the development of a zonal wind oscillation. Whether or not the system is unstable depends on the strength of the vertical diffusion.

\section{Lindzen's parameterization with a continuous spectrum of waves}

We now discuss the generalization of the Lindzen scheme to the case in which the forcing comprises a spectrum of waves over a range of phase speeds. As in the two-wave case, each wave is allowed to affect the mean flow above its initial breaking level, so unlike in the AD99 scheme, multiple breaking levels for a given wave are possible. The total drag is found by summing the drags corresponding to each of the phase speeds. This makes the scheme more expensive to implement than that of AD99. Figure 12 shows the results of a simulation using this scheme. Apart from the intermittency factor, all the parameters used are the same as those in the two-wave simulations, and the momentum flux at the source is given by (4.4). As in section $4, \varepsilon$ is chosen to be proportional to the phase speed resolu- tion; for consistency with the two-wave simulations in which $\varepsilon$ was set to unity, we set $\varepsilon=2 / N_{c}$, where $N_{c}$ is the number of waves in the spectrum. The resulting QBO, shown in Fig. 12a, looks very similar to that obtained in the two-wave case. Figure $12 \mathrm{~b}$ shows the zonal mean wind at the end of the 12-yr run. As before, the black dots show the breaking levels of the waves. With the exception of the small-phase-speed westerly waves $\left(0 \mathrm{~m} \mathrm{~s}^{-1}<c<8 \mathrm{~m} \mathrm{~s}^{-1}\right)$ that break and are completely absorbed close to the source level, each wave has more than one breaking level over the range of heights from the source level to the lid of the computational domain. In the notation of section 3 , we can denote the breaking levels by $z_{b}(c)$ and $z_{b 2}(c)$. For each wave, the upper level of the range of heights in which the wave deposits its momentum is indicated by a circle, and we denote such a level by $z_{\max }(c)$. The arrows show the vertical regions in which momentum is deposited. The black

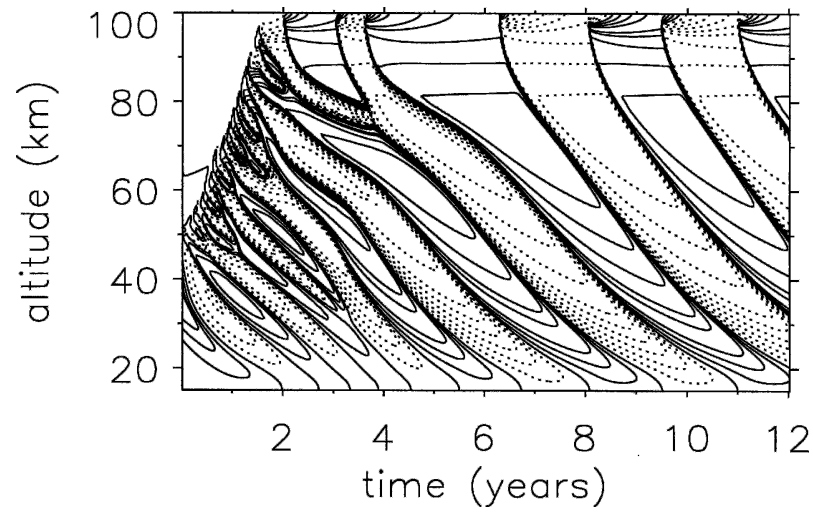

FIG. 11. AD99 parameterization with the momentum flux spectrum shown in Fig. 10a. Time-height plot of zonal mean velocity over a period of $12 \mathrm{yr}$. The solid contours denote westerlies, including the zero-wind line, and the dotted contours denote easterlies. Contour intervals are $5 \mathrm{~m} \mathrm{~s}^{-1}$. 
(a)

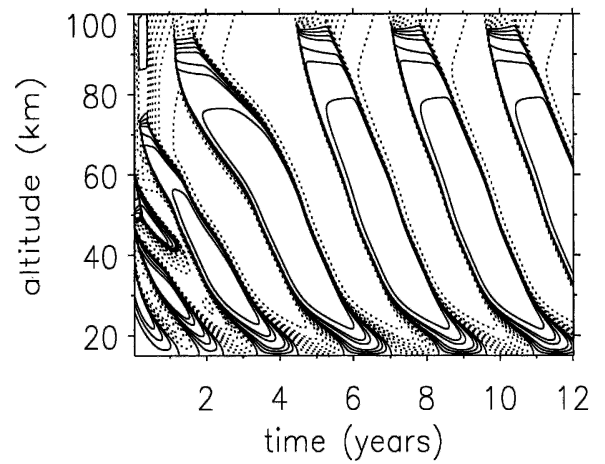

(b)

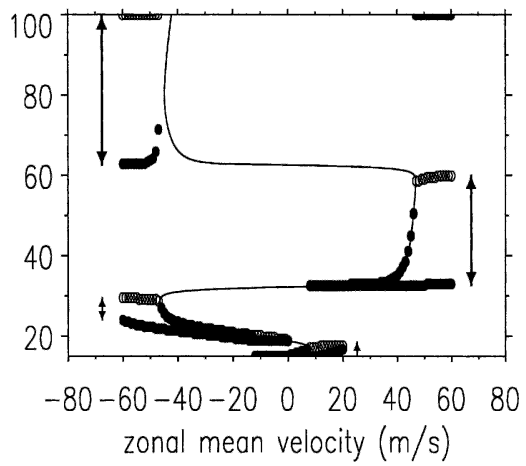

FIG. 12. Lindzen parameterization with a spectrum of waves with the momentum flux spectrum $F_{0}(c)=(\operatorname{sgn} c) \times$ a constant. (a) Time-height plot of $\bar{u}$. The solid contours denote westerlies, including the zero-wind line, and the dotted contours denote easterlies. Contour intervals are $5 \mathrm{~m} \mathrm{~s}^{-1}$. (b) Mean velocity at the end of the 12-yr run. Black dots show the breaking levels $z_{b}(c)$ of the waves; circles show the levels $z_{\max }(c)$. The vertical regions in which the waves deposit their momentum are indicated by the arrows.

dots that lie directly on the $\bar{u}$ curve correspond to breaking levels that are also critical levels. For each wave that has a critical level, the breaking/critical level $z_{b}(c)$ or $z_{b 2}(c)$ also coincides with $z_{\max }(c)$; this means that the black dot coincides with the circle, and the circle is not visible in the graph.

The conclusions of the preceding sections regarding the dependence of the form of the QBO on the input parameters and on the strength of the diffusion apply for this multiple-wave version of the Lindzen scheme as well. A zero initial state with an antisymmetric source spectrum is unstable to small perturbations in the wind or in the momentum flux. In general, a configuration that can generate a $\mathrm{QBO}$ when used in the two-wave Lindzen scheme works for the multiple-wave case as well. But there are some additional features in the multiple-wave case. For example, the drag is in general nonzero from the source level up, because of the waves with small intrinsic phase speed that break at low levels. The shape of the source spectrum affects the drag profile and hence the form of the oscillation, for example, a broad spectrum that includes small-amplitude waves with large intrinsic phase speeds (such as that shown in Fig. 10a) results in more drag at high levels, but this does not create substantial qualitative differences at lower levels.

The main differences between the multiple-wave Lindzen scheme and the AD99 scheme are due to the fact that the former allows higher breaking levels and hence, for a given configuration, it always generates more drag at high levels. Thus, even with a truncated source spectrum such as (4.4), the range of QBO altitudes is from the lowest breaking level (the source level in this case) to the upper boundary of the computational domain. In contrast, the AD99 scheme with the spectrum (4.4) gives a QBO that extends from the source level to a height of about $52 \mathrm{~km}$, as seen in Fig. 7 a.

\section{Discussion}

We have described some of the requirements for simulating a QBO using a simple one-dimensional model with parameterized gravity wave drag. The discussion provides answers to the questions that were posed in section 1 .

There are a number of differences between the gravity wave drag schemes and the HL72 scheme for equatorial planetary waves. In the HL72 scheme, although the strength of the drag, and hence the period of the QBO, depends directly on the amplitude of the waves at their source, the vertical profile of the drag does not depend on the wave amplitude. In the gravity wave drag schemes, on the other hand, the amplitude of the waves determines the levels at which the waves break and thus determines the vertical profile of the drag. The drag profile is, in general, discontinuous in height and the levels where the discontinuities occur are determined by the vertical profile of the mean wind. A local variation in the wind at a given level can have a profound effect on the profile of wave drag at higher levels. In the AD99 scheme, the initial strength of the shear (relative to the other input parameters) is an important factor in determining whether it is possible to generate a mean-wind oscillation at all. In the HL72 planetary wave scheme, on the other hand, local variations in the 
mean wind are not so important, since the drag at any level is determined by the cumulative effect of the wind over the whole range of heights below.

It was seen that, in the AD99 scheme, there is downward propagation of information, even in the absence of vertical diffusion. This is in contrast to the HL72 scheme for equatorial planetary waves, in which there is downward propagation of phase only and the wind evolution is driven by the wind at lower levels (Plumb 1977). In addition, in AD99 configurations with weak wind shear, the evolution of the wind at a given level depends only on the local shear and is independent of the flow evolution at lower levels; in this regime, the scheme thus resembles the LH68 gravity wave drag scheme. In particular, there is downward but no upward propagation of information, and the wind cannot change direction (in this regime). The shear zones, in general, become narrower as they propagate downward, to the extent that a nonoscillating state can sometimes evolve into a state where a mean-wind oscillation is possible. Also, in practice, noise from the numerical evaluation of the drag can destabilize a nonoscillating state and allow the development of a mean-wind oscillation. On the other hand, with sufficiently strong vertical diffusion reducing the strength of the lower level shear zones, the nonoscillating state can be maintained indefinitely.

In all of the schemes discussed here, the strength of the vertical diffusion affects the period and structure of the QBO. Increasing the strength of the diffusion acts to shorten the period. To generate a QBO using the HL72 scheme, vertical diffusion is only needed at the lowest levels, as Plumb (1977) pointed out. With the gravity wave drag schemes that were considered here, however, it is necessary to include vertical diffusion in the vicinity of and above each of the regions of wave momentum deposition. This means that, in practice, diffusion must be included at all levels.

Comparing the different gravity wave drag schemes, it was seen that the AD99 scheme is similar to the two-wave Lindzen scheme in the sense of the drag profile being discontinuous in height (case 2 in section 4), although in the special case where the mean shear is weak (case 1 in section 4), the evolution of the wind is similar to that in the LH68 scheme.

In the AD99 scheme, the width and shape of the source spectrum determine the drag profile and, hence, the form of the QBO (assuming it is possible to generate one). A broad spectrum, including waves of large intrinsic phase speed, results in a wide range of QBO altitudes. With the Lindzen scheme, there are no specific constraints on the width of the source spectrum, since the drag at high levels results from waves any- where within the spectrum that have broken at lower levels, not just from the large-phase-speed waves; in fact, as was shown in section 3, it is possible to generate a QBO with just two waves using the Lindzen scheme. For all the schemes, a zero initial wind profile with an antisymmetric source spectrum is unstable to small perturbations in the wind or the source spectrum for sufficiently weak vertical diffusion.

All the gravity wave parameter settings used here are such that the waves break gravitationally, so they are able to generate QBOs even in the absence of critical levels. This is unrealistic for the terrestrial stratosphere, where gravity wave breaking is mostly due to critical level interactions. However, for our model, which does not include any equatorial planetary wave forcing, it is the only way to generate a mean-wind oscillation from a zero initial wind state. The obvious next step in our investigation is to examine the role of gravity wave drag in the presence of equatorial planetary wave forcing, as this is the case of relevance to the terrestrial atmosphere. In that case, there are additional constraints on the phase speeds of the waves. These are discussed in Campbell and Shepherd (2005).

Acknowledgments. This research was supported by the Natural Sciences and Engineering Research Council of Canada, in part through the Modelling of Global Chemistry for Climate (GCC) project. The authors are grateful to Drs. C. McLandress and M. J. Alexander for helpful discussions, and to Dr. T. J. Dunkerton and an anonymous referee for constructive criticism of the manuscript.

\section{REFERENCES}

Alexander, M. J., and T. J. Dunkerton, 1999: A spectral parameterization of mean-flow forcing due to breaking gravity waves. J. Atmos. Sci., 56, 4167-4182.

Andrews, D. G., J. R. Holton, and C. B. Leovy, 1987: Middle Atmosphere Dynamics. Academic Press, 489 pp.

Baldwin, M. P., and Coauthors, 2001: The quasi-biennial oscillation. Rev. Geophys., 39, 179-229.

Booker, J. R., and F. P. Bretherton, 1967: The critical level for gravity waves in a shear flow. J. Fluid Mech., 27, 513-539.

Campbell, L. J., and T. G. Shepherd, 2005: Constraints on wave drag parameterization schemes for simulating the quasibiennial oscillation. Part II: Combined effects of gravity waves and equatorial planetary waves. J. Atmos. Sci., 62, 4196-4205.

Dunkerton, T. J., 1981a: Wave transience in a compressible atmosphere. Part I: Transient internal wave, mean-flow interaction. J. Atmos. Sci., 38, 281-297.

, 1981b: Wave transience in a compressible atmosphere. Part II: Transient equatorial waves in the quasi-biennial oscillation. J. Atmos. Sci., 38, 298-307.

_ 1982: Wave transience in a compressible atmosphere. Part 
III: The saturation of internal gravity waves in the mesosphere. J. Atmos. Sci., 39, 1042-1051.

_ 1991 : Nonlinear propagation of zonal winds in an atmosphere with Newtonian cooling and equatorial wavedriving. J. Atmos. Sci., 48, 236-263.

_ 1997: The role of gravity waves in the quasi-biennial oscillation. J. Geophys. Res., 102, $26053-26076$.

- 2000: Inferences about QBO dynamics from the atmospheric "tape recorder" effect. J. Atmos. Sci., 57, 230-246.

Ebdon, R. A., 1960: Notes on the wind flow at $50 \mathrm{mb}$ in tropical and subtropical regions in January 1957 and in 1958. Quart. J. Roy. Meteor. Soc., 86, 540-542.

Giorgetta, M. A., E. Manzini, and E. Roeckner, 2002: Forcing of the quasi-biennial oscillation from a broad spectrum of atmospheric waves. Geophys. Res. Lett., 29, 1245, doi: 10.1029/2002GL014756.

Gray, L. J., and J. A. Pyle, 1989: A two-dimensional model of the quasi-biennial oscillation of ozone. J. Atmos. Sci., 46, 203220 .

Hamilton, K., R. J. Wilson, and R. Hemler, 1999: Middle atmosphere simulated with high vertical and horizontal resolution versions of a GCM: Improvement in the cold pole bias and generation of a QBO-like oscillation in the tropics. J. Atmos. Sci., 56, 3829-3846.

Holton, J. R., 1982: The role of gravity wave induced drag and diffusion in the momentum budget of the mesosphere. J. Atmos. Sci., 39, 791-799.

— biennial cycle of the tropical stratosphere. J. Atmos. Sci., 29, 1076-1080.

Horinouchi, T., and S. Yoden, 1998: Wave-mean flow interaction associated with a QBO-like oscillation in a simplified GCM. J. Atmos. Sci., 55, 502-526.

- and Coauthors, 2003: Tropical cumulus convection and upward propagating waves in middle atmospheric GCMs. J. Atmos. Sci., 60, 2765-2782.

Lindzen, R. S., 1981: Turbulence and stress owing to gravity wave and tidal breakdown. J. Geophys. Res., 86, 9707-9714.

- and J. R. Holton, 1968: A theory of the quasi-biennial oscillation. J. Atmos. Sci., 25, 1095-1107.

McFarlane, N. A., 1987: The effect of orographically excited gravity wave drag on the general circulation of the lower stratosphere and troposphere. J. Atmos. Sci., 44, 1775-1800.

McLandress, C., 2002: Interannual variations of the diurnal tide in the mesosphere induced by a zonal-mean wind oscillation in the tropics. Geophys. Res. Lett., 29, 1305, doi:10.1029/ 2001 GL014551.

Plumb, R. A., 1977: The interaction of two internal waves with the mean flow: Implications for the theory of the quasi-biennial oscillation. J. Atmos. Sci., 34, 1847-1858.

Reed, R. J., W. J. Campbell, L. A. Rasmussen, and R. G. Rogers, 1961: Evidence of a downward propagating annual wind reversal in the equatorial stratosphere. J. Geophys. Res., 66, 813-818.

Scaife, A. A., N. Butchart, C. D. Warner, D. Stainforth, W. Norton, and J. Austin, 2000: Realistic quasi-biennial oscillation in a simulation of the global climate. Geophys. Res. Lett., 27, 3481-3484.

Takahashi, M., 1996: Simulation of the stratospheric quasibiennial oscillation using a general circulation model. Geophys. Res. Lett., 23, 661-664. 